\title{
Role of iron in pathogenesis of Parkinson disease
}

\author{
JOLANTA GAŁAZZKA-FrIEDMAN ${ }^{1}$, ANDRZEJ FRIEDMAN ${ }^{2 *}$ \\ ${ }^{1}$ Faculty of Physics, Warsaw University of Technology \\ ${ }^{2}$ Department of Neurology, Medical University in Warsaw \\ * Corresponding author: andrzej.friedman@wum.edu.pl
}

\begin{abstract}
Parkinson's disease is one of the most frequent human neurodegenerations. Motor symptoms of Parkinson's disease are the consequence of the destruction of nervous cells in the substantia nigra (SN), a small (about $500 \mathrm{mg}$ ) structure located deep in human brain. The concentration of iron in SN is comparable to that in liver and is equal to about $180 \pm 60 \mathrm{ng} / \mathrm{mg}$ of wet tissue and the iron in $\mathrm{SN}$ is mostly bound to ferritin. For many years it has been believed that the degeneration of nervous cells in SN in Parkinson's disease is related to an important increase in the concentration of iron. Our own studies based on Mössbauer spectroscopy and other studies conducted with the use of various techniques have not confirmed this finding. The ratio of the concentration of iron in PD vs. control SN evaluated by Mössbauer spectroscopy was found to be equal $1.00 \pm 0.13$. We also confirmed that most of iron in SN is located within ferritin. ELISA studies demonstrated a significant decrease in L ferritin in parkinsonian SN compared to the control group. As L-ferritin is related to safe keeping of iron within the ferritin shell, its decrease may lead to an efflux of iron and increase in the concentration of labile iron. Indeed our studies did show a difference in the concentration of labile iron between PD and control SN ( $135 \pm 10 \mathrm{ng} / \mathrm{g} \mathrm{vs.} 76 \pm 5 \mathrm{ng} / \mathrm{g}$ ). This labile iron, which may initiate Fenton reaction, may be the cause of the oxidative stress leading to the death of nervous cells in PD.
\end{abstract}

Key words: iron, Parkinson disease, oxidative stress, Mössbauer spectroscopy, electron microscopy, ELISA

\section{Introduction}

Parkinson's disease (PD) is a neurological disorder with its prevalence rising with the age of the population. While in the population as a whole the prevalence is about $0.15 \%$, in the population over 65 years it is $1.5 \%$ (Koller et al., 1986). The main clinical features of the disease include slowness of movements (bradykinesia), rigidity of muscles and rest tremor (Litvan et al., 2003). These symptoms are due to the progressive degeneration of substantia nigra (SN), a small structure of about $500 \mathrm{mg}$ located bilaterally deep in the brain in mesencephalon. The death of nervous cells in SN causes a dramatic decrease in the concentration of dopamine in other brain structures - globus pallidus (GP) and putamen. SN, GP, putamen together with some other brain structures form the so called extrapyramidal system. The discovery that parkinsonian symptoms are due to the decrease in dopamine levels, made by Carlsson (Carlsson et al., 1957), was awarded the Nobel Prize in 2000. The first symptoms of PD appear after the death of about $50 \%$ of the nervous cells in SN. This destruction of a half of SN causes a decrease in the concentration of dopamine whose level at the onset of the symptoms equals $20 \%$ of the normal value.

The very name of this structure - substantia nigra (black structure) comes from the presence of a black pigment named neuromelanin which is normally found in dopaminergic cells in SN. In PD, the nervous cells containing neuromelanin disappear and $\mathrm{SN}$ becomes pale.

The cause of the death of nervous cells in PD remains unknown, although there are several hypotheses that explain it. Certainly, genetic and environmental factors are involved, and both may trigger the death of nervous cells via various mechanisms. One of these is oxidative stress.

Oxidative stress is the condition, when the production of free radicals surpasses their inactivation. Free radicals which are not scavenged in time may cause the death of nervous cells through peroxidation of lipids in cells membranes or by direct lesions to DNA. Oxidative stress is initiated by a chemical reaction referred to as the Fenton reaction: 


$$
\mathrm{Fe}^{2+}+\mathrm{H}_{2} \mathrm{O}_{2} \rightarrow \mathrm{Fe}^{3+}+\mathrm{OH}^{\cdot}+\mathrm{OH}^{-}
$$

The hydroxyl free radical $\left(\mathrm{OH}^{*}\right)$ is $10^{14}$ times more active than the hydroxyl ion $\left(\mathrm{OH}^{-}\right)$. It is very difficult to prove an overproduction of free radicals due to their very short half-life and the oxidative stress theory is based on indirect proofs, such as a decrease in the concentration of free radicals scavengers or an increase in malonic aldehyde (which is a product of lipids peroxidation).

The hypothesis that the increase in the concentration of iron in SN is the main cause of PD was formulated in 1989. Some authors named Parkinson's disease “a progressive siderosis of SN” (Youdim et al., 1989). This hypothesis was based on the results of several studies, where a significant increase in the concentration of the total iron in SN in PD compared to the control was observed. One of these studies used spectrophotometry to assess the concentration of iron (Sofic et al., 1988). In this study, apart from an $80 \%$ increase in the concentration of iron in parkinsonian SN compared to the control, an important concentration of divalent iron was also found. The authors did not identify any iron-containing compounds in SN.

The initial goal of our Mössbauer studies was to identify the iron-binding compounds present in $\mathrm{SN}$ and to verify the results of the spectrophotometric studies concerning the changes in the $\mathrm{Fe}^{2+} / \mathrm{Fe}^{3+}$ ratio and the changes in the concentration of total iron present in parkinsonian SN compared to the control SN.

Mössbauer Spectroscopy has several advantages for investigating the role of iron in the pathogenesis of Parkinson's disease:

- there is no oxidation or spin state of iron which is Mössbauer silent;

- it is the only technique, which does not need any sample preparation, except the freezing of the sample;

- it distinguishes unequivocally between divalent and trivalent iron;

- it may identify iron-binding compounds;

- it can assess the iron concentration in a sample if it is higher than about $10 \mu \mathrm{g} / \mathrm{cm}^{3}$.

Later in this paper, we will present our own studies on the role of iron in the pathogenesis of $\mathrm{PD}$, performed with the use of not only of Mössbauer spectroscopy but also electron microscopy, ELISA and atomic absorption.

\section{The question of the choice of samples for investigation}

The method of storage of human tissues, prepared for scientific research, must be adapted to the scientific problem which is to be addressed. Presently, there are two ways of storing brain tissues: conservation in formalin or storage at low temperatures.

The advantage of storage of tissue in formalin is the preservation of the cellular membranes. Storage at low temperatures destroys the cellular membrane, but quite effectively blocks chemical processes.

Preserving human tissues in formalin began in the second half of the $19^{\text {th }}$ century. In some scientific centers there are banks of brain tissues stored since that time. Freezing of brain tissues started in the second half of the $20^{\text {th }}$ century.

At the beginning of our studies, we used samples which had been stored in formalin (Bauminger et al., 1994; Galazka-Friedman et al., 1996) as these samples were readily available. The first comparative studies were performed on samples which had been stored in formalin and were later lyophilized. In the next step, samples which did not have any contact with formalin were measured. Comparison of the Mössbauer spectra of these two types of samples are discussed in a paper by Bauminger and coworkers (1994). Mössbauer spectra usually show a characteristic symmetric doublet for each dia- or para-magnetic compound and a characteristic sextet for each magnetic compound. All Mössbauer spectra obtained at $90 \mathrm{~K}$ present only one doublet. Significant differences were observed in the measurements performed at $4.1 \mathrm{~K}$. The Mössbauer spectra of the samples which were not stored in formalin but were frozen immediately after the autopsy, presented only one sextet at $4.1 \mathrm{~K}$. The Mössbauer spectra of samples which were stored in formalin for a longer time, obtained at $4.1 \mathrm{~K}$, were composed of one sextet and one doublet.

The parameters of the sextet at $4.1 \mathrm{~K}$ and the doublet at $90 \mathrm{~K}$ are very similar to those observed in ferritin or hemosiderin. The spectra observed in the isolated iron-storage proteins of ferritin or hemosiderin are determined by the unique magnetic properties of these proteins, in which the iron cores of the proteins are antiferromagnetically ordered, but display the phenomenon of superparamagnetism because of the small size of the iron cores. At $4.1 \mathrm{~K}$ in ferritin, a well-defined six-line 
Mössbauer spectrum is observed, corresponding to an internal magnetic field. At higher temperatures, a quadrupole doublet replaces this spectrum and the magnetic interaction is averaging to zero because of superparamagnetic fluctuations in small nanoparticles.

Further systematic Mössbauer studies of the samples of SNs showed that the intensity of the doublet obtained at $4.1 \mathrm{~K}$ is not a marker of the disease, but depends on the time of storage of the samples in formalin (Bauminger et al., 1994; Galazka-Friedman et al., 1996). It is possible that the ferritin protein was denatured during the long-term storage in formaldehyde enabling an efflux of iron from the protein shell yielding the doublet in the $4.1 \mathrm{~K}$ spectra.

It was clear from these studies that the most proper form of storage for samples needed for our studies is storage at low temperatures, without any contact with formalin. And such samples were chosen for later studies.

Mössbauer studies described in the next part of this paper were performed on $\mathrm{SNs}$ which were isolated, 48 hours after death, with plastic knives. Samples for Mössbauer studies, each consisting of one $\mathrm{SN}$, were placed in plastic holders, frozen, and stored in liquid nitrogen until measurements. The second $\mathrm{SN}$ from the same brain was used for histopathological studies to verify the diagnosis of PD.

\section{Concentration of divalent iron in SN}

For several reasons, Mössbauer spectroscopy is the best method to determine the $\mathrm{Fe}^{2+} / \mathrm{Fe}^{3+}$ ratio (Bancroft, 1973), inter alia the fact that (i) there is a large difference in Mössbauer spectra and parameters between high spin $\mathrm{Fe}^{2+}$ and $\mathrm{Fe}^{3+}$, and (ii) that the samples to be investigated by Mössbauer spectroscopy do not need any chemical pretreatment for measurements, which minimizes the possibility of oxidation or reduction of iron within the samples during their preparation.

In our Mössbauer studies, we could not detect any signal from divalent iron. Therefore, in order to estimate the amount of divalent iron, which could nevertheless be present in a sample, we performed a computer simulation. We included a doublet with Mössbauer parameters typical for $\mathrm{Fe}^{2+}, \mathrm{IS}=1.23 \mathrm{~mm} / \mathrm{s}, \mathrm{QS}=2.96 \mathrm{~mm} / \mathrm{s}$, in the theoretical spectra that were computer fitted to the experimentally observed spectra. The quality of the computer fits (as measured by the chi-square) did not change significantly if a $\mathrm{Fe}^{2+}$ doublet, whose intensity was up to $5 \%$ of the overall spectral area, was included in the theoretical spectra. This value is an upper limit for the percentage of divalent iron that could be present in the samples and could not be detected by us in our Mössbauer experiments (Galazka et al., 1996) This result should be compared with the ratios of $\mathrm{Fe}^{2+} / \mathrm{Fe}^{3+}$ determined by spectrophotometry, which were 3:1 for control SN and 1:1 for parkinsonian SN (Sofic et al., 1988).

To understand this difference between Mössbauer and spectrophotometric data, the spectrophotometric experiment described by Sofic and coworkers (1988) was repeated in our laboratory. In that experiment, we could also identify a large amount of divalent iron. This could be explained by the way in which the samples were prepared by the authors of the cited paper: the investigated tissues were homogenized in the presence of pepsin and $\mathrm{HCl}$. In samples prepared in such a way the protein shell of ferritin gets destroyed and trivalent iron may be reduced.

\section{Identification of iron-binding compounds present in substantia nigra}

For identification of the iron-binding compounds present in SN, Mössbauer measurements of control and parkinsonian samples were performed at temperatures of $297 \mathrm{~K}, 90 \mathrm{~K}, 4.1 \mathrm{~K}$. For these studies all samples were frozen shortly after autopsy. Some of the samples were also lyophilized. Mössbauer measurements of tissues at room temperature can be performed only on lyophilized samples. Mössbauer spectra of SN (control as well as parkinsonian) obtained at $297 \mathrm{~K}$ and $90 \mathrm{~K}$ showed only one doublet, while Mössbauer spectra obtained at 4.1 showed only one sextet.

The transformation of Mössbauer spectra of SNs as a function of decreasing temperature from a doublet at $297 \mathrm{~K}$ and $90 \mathrm{~K}$ to a sextet at $4.1 \mathrm{~K}$, suggests that almost all iron atoms in SN form clusters and interact magnetically at low temperatures. The above-presented evolution of the Mössbauer spectra as the function of temperature is a characteristic for spectra of small nanoparticles, in which the iron orders antiferromagnetically, but displays the phenomenon of superparamagnetism because of the small size of these particles. This is especially the case for ferritin or hemosiderin, where the size of iron cores is smaller than $7 \mathrm{~nm}$. The Mössbauer parameters of the doublet at $90 \mathrm{~K}$ and at room temperature, 
and the Mössbauer parameters of the sextet at $4.1 \mathrm{~K}$ are also very similar to those observed for ferritin or hemosiderin (Galazka-Friedman et al., 1996). This result strongly suggests that iron in SNs is bound mainly to ferritin or hemosiderin.

Youdim and collaborators tried to detect ferritin in SNs (Ben-Shachar and Youdim, 1992) by electron microscopy which provided negative results as the iron cores of ferritin or hemosiderin were not observed.

To solve this controversy we also used electron microscopy. Samples for electron microscopy were prepared from ferritin and hemosiderin isolated from the brain (substantia nigra, globus pallidus, hippocampus) and the liver. Samples for electron microscopy of the whole tissue of SN were also prepared (Galazka-Friedman et al., 1998, Friedman and Galazka-Friedman, 2001).

The average diameter of the iron cores of ferritin in SN tissues was evaluated as $3.8 \pm 0.5 \mathrm{~nm}$ and that of the iron cores of ferritin isolated from SN was found to be $3.7 \pm 0.5 \mathrm{~nm}$. The average diameter of the iron cores of ferritin present in the brain turned out to be much smaller than that found for ferritin isolated from the liver (6.5 $\pm 0.5 \mathrm{~nm})$.

Measurements of iron cores of hemosiderin isolated from SN showed that iron cores of hemosiderin isolated from this part of the brain are much smaller (2.0 $\pm 0.5 \mathrm{~nm}$ ) than those of the iron cores of hemosiderin present in other parts of the human body. In the spleen e.g., the size of the iron cores of hemosiderin is about $3.5 \mathrm{~nm}$ (Bell et al., 1984).

The cited results obtained by Mössbauer spectroscopy and electron microscopy support the hypothesis that iron in SN is mainly bound to ferritin and hemosiderin. Difficulties to observe iron cores of ferritin in SN reported by some authors (Iancu, Ben-Shachar, Hill, Youdim, unpublished data) were probably caused by the much smaller size of these iron cores compared to the well-known size of iron cores of ferritin present in the liver. The structure and function of ferritin in relation to Parkinson's disease have been recently discussed (Friedman et al., 2011).

Some authors have suggested that iron in SN may be bound mainly to neuromelanin (NM) (Jellinger et al., 1992). Computer simulations (Galazka-Friedman et al., 1996; Galazka-Friedman et al., 2004) demonstrated that if less than $15 \%$ of the iron in SN was bound to NM, it could escape detection in our measurements. Such simu- lations were done for synthetic neuromelanin (Galazka-Friedman et al., 1996) and for neuromelanin isolated from SN (Galazka-Friedman et al., 2004).

\section{Comparison of the amount of iron in control and parkinsonian $\mathrm{SN}$ - total and labile iron}

Efforts to estimate the concentration of iron in parkinsonian SNs and control SNs were conducted in different laboratories, using different techniques. The results obtained were controversial. In the studies conducted by Galazka-Friedman and Friedman (1997), the reasons for these discrepancies were analyzed. In the following section, some examples are cited. The concentrations of iron in different parts of the brain were determined for the first time by Hallgren and Sourander in 1958 by colorimetry. The results obtained in this experiment $(184.6 \pm 65.2 \mu \mathrm{g} / \mathrm{g}$ wet tissue) are in agreement with our Mössbauer data $-177 \pm 14 \mu \mathrm{g} / \mathrm{g}$ wet tissue for the control and $177 \pm 18 \mu \mathrm{g} / \mathrm{g}$ wet tissue for PD (Galazka-Friedman et al., 1996, Wypijewska et al., 2010).

The first comparison of iron concentrations in parkinsonian and the control brains was performed by Earle (Earle, 1968), who employed the X-ray fluorescence (XRF) method. In this study, a 2-fold increase in the iron level in parkinsonian brains as compared to the control was observed. These measurements were done without calibration, so absolute values for the amount of iron are not available. In addition, no errors were reported. In this paper the described experiments were performed on a large number of samples. Because of this, Earle's paper is cited as a very important source of information. Looking for possible reasons for such a large discrepancy with our Mössbauer results, it should be noted that Earle had studied samples stored in formalin for more than 70 years. This fact could be the reason for such a high ratio between the concentration of iron in parkinsonian SN and the control SN. It is possible that more iron leaked from ferritin in the control SNs compared to the control SNs that were stored in formalin much longer than parkinsonian SNs.

In 1988 (Sofic et al., 1988), the concentration of iron in control and parkinsonian SNs was evaluated by spectrophotometry. The number of control and parkinsonian SN samples was the same $(n=8)$. The weight of the investigated samples was between 50 and $80 \mathrm{mg}$. The average concentration of iron in control samples was $48 \pm 8 \mu \mathrm{g} / \mathrm{g}$ wet tissue and in parkinsonian samples $85 \pm 11 \mu \mathrm{g} / \mathrm{g}$ wet 
tissue. The concentration of iron in parkinsonian SN compared to control $\mathrm{SN}$ was $1.77 \pm 0.37$. This experiment seems to support the argument about the increase in iron in parkinsonian SN compared to the control SN.

The absolute concentration of iron found in that experiment (Sofic et al., 1988) was much smaller than in our Mössbauer data (3.6 times for control samples and 3.1 times smaller for parkinsonian samples). The concentration of iron in the control tissues determined by spectrophotometry was also 3.8 times lower than the concentration of iron determined by colorimetry (Hallgren and Sourander, 1958). It should be noted that the discussed experiment was the same experiment in which a large percentage of divalent iron was found. Such high amount of divalent iron could only be an artifact, caused by the special method of preparing samples needed for spectrophotometry. The presence of divalent iron in the samples was most probably the result of the reduction of trivalent iron released from ferritin. The lower concentration of total iron found in that experiment could be due to the migration of iron to the supernatant. In other experiments (Zecca et al., 2001, Zecca et al., 2005), the concentration of iron was found to be similar to that obtained by colorimetry (Hallgren and Sourander, 1958) and in Mössbauer measurements.

Although there is no evidence of an increase in the concentration of total iron in parkinsonian SN compared to the control SN, a significant increase in the concentration of the labile, non ferritin bound iron in PD has been observed (Wypijewska et al., 2010). The concentration of this iron obtained by atomic absorption in PD SN was $135 \pm 10 \mathrm{ng} / \mathrm{g}$ wet tissue, while in the control group it was $76 \pm 5 \mathrm{ng} / \mathrm{g}$ wet tissue. It should be noted that the concentration of the labile iron is about 2000 times lower than the concentration of the total iron.

\section{ELISA studies of ferritin in parkinsonian and control substantia nigra}

The structure of ferritin in parkinsonian and the control SN was assessed with the use of enzyme-linked immuno-sorbent assay (ELISA), in which monoclonal antibodies (a generous gift of Professor Arosio) were used (Galazka-Friedman et al., 2004). A significant difference was found between PD and the control group. In the preclinical phase of PD - so called Incidental Lewy Bodies, there is a significant decrease in $\mathrm{L}$ ferritin $(50.6$ $\pm 4.7 \mathrm{ng} / \mathrm{mg}$ wet tissue vs. $98 \pm 12 \mathrm{ng} / \mathrm{mg}$ wet tissue), with the concentration of $\mathrm{H}$ ferritin similar to the control $(336.9 \pm 35.8 \mathrm{ng} / \mathrm{mg}$ wet tissue). In full-blown PD, the significant decrease of $\mathrm{L}$ ferritin compared to the control 52 $\pm 8 \mathrm{ng} / \mathrm{mg}$ vs. $98 \pm 12 \mathrm{ng} / \mathrm{mg}$ is accompanied by an increase in $\mathrm{H}$ ferritin $(534 \pm 71 \mathrm{ng} / \mathrm{mg}$ vs. $375 \pm 38 \mathrm{ng} / \mathrm{mg}$ ) (Koziorowski et al., 2007). As L ferritin plays a crucial role in safe storage of iron within the protein shell of ferritin, a decrease in L ferritin in PD may predispose to an efflux of iron from the iron core. This iron may get reduced to divalent iron and can trigger Fenton reaction leading to oxidative stress.

\section{Conclusions and summary}

From the above-described experimental studies on the role of iron in the pathogenesis of Parkinson's disease the following conclusions can be formulated:

- iron present in parkinsonian and in control $\mathrm{SN}$ is bound mainly to ferritin or hemosiderin;

- in Mössbauer spectra of SNs no signal from divalent iron is detected. Taking into consideration the experimental limit, the upper limit for the concentration of divalent iron in SN can only be less than 5\% of all iron in $\mathrm{SN}$;

- Mössbauer studies did not confirm an increase in the concentration of iron in parkinsonian SN compared to the control;

- in PD there is a significant increase in the labile nonferritin bound iron in SN;

- in preclinical PD there is a significant decrease in $\mathrm{L}$ ferritin in SN, which later on in full blown disease is accompanied by an increase in $\mathrm{H}$ ferritin.

The presented data suggest that the mechanism of nervous cells death in Parkinson's disease may depend on an increase in the labile iron which escapes from the ferritin shell due to its change of the structure leading to an inability to keep iron safely inside.

\section{References}

Bancroft G.M. (1973) Mössbauer spectroscopy. An introduction for inorganic chemists and geochemists. McGraw Hill Book Company, London.

Bauminger E.R., Barcikowska M., Friedman A., Gałązka-Friedman J., Hechel D., Nowik I. (1994) Does iron play a role in Parkinson's disease? Hyperfine Interact. 91: 853-856.

Bell S.H., Weir M.P., Dickson D.P.E., Gibson J.I., Sharp G.A., Peters T.J. (1984) Mössbauer spectroscopic studies of 
human haemosiderin and ferritin. Biochim. Biophys. Acta 787: 227-236

Ben-Shachar D., Youdim M.B.H. (1992) Brain iron and nigrostriatal dopamine neurons in Parkinson's disease. [in:] Iron and human disease, ed. Lauffer R.B., CRC Press Inc., Boca Raton, p: 349-363.

Carlsson A., Lindqvist M., Magnusson T. (1957) 3,4-Dihydroxyphenylalanine and 5-hydroxytryptophan as reserpine antagonists. Nature 180: 1200-1202.

Earle K.M. (1968) Studies on Parkinson's disease including $x$ ray fluorescent spectroscopy of formalin fixed brain tissue. J. Neuropathol. Exp. Neurol. 27: 1-13.

Friedman A., Gałązka-Friedman J. (2001) The current state of free radicals in Parkinson's disease: nigral iron as a trigger of oxidative stress. Adv. Neurol. 86: 137-142.

Friedman A., Arosio P., Finazzi D., Koziorowski D., GalazkaFriedman J. (2011) Ferritin as an important player in neurodegeneration. Park and Rel. Disord. doi:10.1016/ j.parkreldis.2011.03.016.

Gałązka-Friedman J., Bauminger E.R., Friedman A., Barcikowska M., Hechel D., Nowik I. (1996) Iron in parkinsonian and control substantia nigra - a Mössbauer spectroscopy study. Movement Dis. 11: 8-16.

Gałąka-Friedman J., Friedman A. (1997) Controversies about iron in parkinsonian and control substantia nigra. Acta Neurobiol. Exp. 57: 210-225.

Gałązka-Friedman J., Bauminger E.R., Tymosz T., Friedman A. (1998) Mössbauer spectroscopy, electron microscopy and electron diffraction studies of ferritin-like iron in human heart, liver and brain. Hyperfine Interact. (C) 3 : 49-52.

Galazka-Friedman J., Bauminger E.R., Koziorowski D., Friedman A. (2004) Mössbauer spectroscopy and ELISA studies reveal differences between Parkinson's disease and control substantia nigra. Biochim. Biophys. Acta 168: 130-136.

Hallgren B., Sourander P. (1958) The effect of age on the nonhaemin iron the human brain. J. Neurochem. 3: 41-51.

Jellinger K., Kienzel E., Rumpelmair G., Riederer P., Stachelberger H., Ben-Shachar D., Youdim M.B.H. (1992) Ironmelanin complex in substantia nigra of parkinsonian brains. an X-ray microanalysis. J. Neurochem. 59: 1168-1171.
Koller W.C., O’Hara R., Weiner W., Lang A.E., Nutt J., Agid Y., Bonnet A.M., Jankovic J. (1986) Relationship of aging to Parkinson's disease. Adv. Neurol. 45: 317-321.

Koziorowski D., Friedman A., Arosio P., Santambrogio P., Dziewulska D. (2007) ELISA reveals a difference in the structure of substantia nigra ferritin in Parkinson's disease and Incidental Lewy Body compared to control. Park. Rel. Disord. 13: 214-218.

Litvan I., Bhatia K.P., Burn D.J., Goetz C.C., Lang A.E., McKeith I., Quinn N., Sethi K.D., Shults C., Wenning G.K. (2003) SIC Task Force appraisal of clinical diagnostic criteria for parkinsonian disorders. Mov. Disord. 18: 467-486.

Sofic E., Riederer P., Heinsen H., Beckmann H., Reynolds G.P., Hebenstreit G., Youdim M.B.H. (1988) Increased iron (III) and total iron content in post mortem substantia nigra of parkinsonian brain. J. Neural. Trans. 74: 199-205.

Wypijewska A., Galazka-Friedman J., Bauminger E.R., Wszolek Z.K., Schweitzer K.J., Dickson D.W., Jaklewicz A., Elbaum D., Friedman A. (2010) Iron and reactive oxygen species activity in parkinsonian substantia nigra. Park. Rel. Disord. 16: 329-333.

Youdim M.B.H., Ben-Shachar D., Riederer P. (1989) Is Parkinson's disease a progressive siderosis of substantia nigra resulting in iron and melanin induced neurodegeneration? Acta Neurol. Scand. 126: 47-54.

Zecca L., Gallorini M., Schuenemann V., Trautwein A.X., Gerlach M., Riederer P., Vezzoni P., Tampellini D. (2001) Iron, neuromelanin and ferritin content in the substantia nigra of normal subjects at different ages: consequences for iron storage and neurodegenerative processes. J. Neurochem. 76: 1766-1773.

Zecca L., Berg D., Arzberger T., Ruprecht P., Rausch W.D., Musicco M., Tampellini D., Riederer P., Gerlach M., Becker G. (2005) In vivo detection of iron and neuromelanin by transcranial sonography: a new approach for early detection of substantia nigra damage. Mov. Disord. 10: 1278-1285. 